\title{
ORIGINAL
}

\section{YODOPOVIDONA EN PLEURODESIS QUÍMICA EN PACIENTES CON DERRAME PLEURALY NEUMOTÓRAX ESPONTÁNEO: SERIE DE CASOS}

\section{YODOPOVIDONE IN CHEMICAL PLEURODESIS IN PATIENTS WITH PLEURAL EFUSSION AND SPONTANEOUS NEUMOTÓRAX: CASE SERIES}

Rafael Alberto Velandia Mahecha, ${ }^{1, a}$, Isabel Cristina Brito Rojas ${ }^{2, a}$

\section{RESUMEN}

Introducción: La pleurodesis química adhiere la pleura para prevenir colapsos pulmonares, el resultado benéfico del procedimiento como tratamiento se determina de acuerdo a si existe o no de derrame sintomático $\circ$ neumotórax. Objetivo: Describir el porcentaje de derrame pleural o neumotórax espontáneo recidivante en sujetos mayores de 18 años que fueron llevados a pleurodesis con Yodopovidona en un Hospital Universitario de tercer de nivel en Bogotá. Métodos: Estudio descriptivo de serie de casos. Se estudiaron 30 pacientes con derrame pleural y neumotórax espontáneo que fueron llevados a pleurodesis utilizando diferentes técnicas entre enero de 2011 y Mayo de 2014 en un Hospital Universitario de tercer nivel en Bogotá. Se incluyeron en el estudio todos los pacientes mayores de 18 años quienes presentaban las patologías arriba descritas y a quienes se realizó pleurodesis química con Yodopovidona. Se excluyeron pacientes menores de 18 años y a quienes se realizó pleurodesis por otras técnicas o con otras sustancias. 14 pacientes cumplían los criterios de inclusión. Los datos fueron recolectados mediante la revisión de historias clínicas. El análisis estadístico se realizó mediante tablas de frecuencias, cálculo de medidas de tendencia central y porcentajes. Resultados: El promedio de edad fue de 61,85 años. El 57,14\% de los pacientes presentaba derrame pleural maligno. Una respuesta completa a la pleurodesis en seguimiento al mes se presentó en 13 de los pacientes evaluados. Como único efecto adverso al procedimiento se reportó dolor. El promedio de estancia hospitalaria fue de 15 días. Conclusión: Basados en el presente estudio y otros se puede considerar que la Yodopovidona es una agente seguro, eficaz, de fácil consecución y bajo costo para la realización de pleurodesis química.

\section{ABSTRACT}

Introduction: Chemical pleurodesis adheres the pleura to prevent pulmonary collapses, the beneficial result of the procedure as a treatment is determined according to whether or not there is a symptomatic effusion or pneumothorax. Objective: To describe the percentage of pleural effusion or recurrent spontaneous pneumothorax in subjects older than 18 years who were taken to pleurodesis with iodopovidone in a University Hospital of third level in Bogotá. Methods: Descriptive study of case series. We studied 30 patients with pleural effusion and spontaneous pneumothorax who were taken to pleurodesis using different techniques between January 2011 and May 2014 in a third level University Hospital in Bogotá. All patients older than 18 years who presented the pathologies described above and who underwent chemical pleurodesis with Yodopovidona were included in the study. We excluded patients under 18 years and who underwent pleurodesis by other techniques or with other substances. 14 patients met the inclusion criteria. The data was collected by reviewing medical records. The statistical analysis was performed using frequency tables, calculation of central tendency measures and percentages.Results: The average age was 61,85 years. 57,14\% of the patients presented malignant pleural effusion. A complete response to pleurodesis in a one-month follow-up was presented in 13 of the patients evaluated. Pain was reported as the only adverse effect. The average length of hospital stay was I5 days. Conclusion: Based on the present study and others, it can be considered that Yodopovidona is a safe, effective, easy to obtain and low cost agent for the performance of chemical pleurodesis.
' Cirujano General - Intensivista ${ }^{2}$ Médico General

anniversidad Militar Nueva Granada

Correspondencia a: Isabel Cristina Brito Rojas Correo: iscribriro@hotmail.com Teléfono: 3163538405

Dirección: Calle 24 sur \# 40a-38 Bogotá, Colombia

Palabras clave: Pleurodesia, Yodopovidona, derrame pleural, neumotórax.

Keywords: Pleurodesia, iodopovidone, pleural effusion, pneumothorax.

Procedencia y arbitraje: no comisionado, sometido a arbitraje externo.

Recibido para publicación: 19 de octubre de 2018 Aceptado para publicación: 29 de julio de 2019

Citar como: Rev Cient Cienc Med 2019;22(1): 17-23 


\section{INTRODUCCIÓN}

Ce define como neumotórax espontáneo al $\checkmark$ colapso de uno o ambos pulmones, causado por la entrada de aire en la cavidad pleural ${ }^{1}$, se estima una incidencia de dicha patología de 7,4-28 casos/100 000 habitantes en hombres y de 1,2-10 casos/100 000 habitantes en mujeres ${ }^{2}$, así mismo pacientes con diagnóstico de enfermedades neoplásicas presentan incidencia de derrames pleurales del $13 \%{ }^{3}$, siendo el cáncer de pulmón la causa más común; seguido del cáncer de seno, gástrico y de ovario ${ }^{4}$. La pleurodesis química es un procedimiento que tiene como finalidad adherir la pleura para evitar colapsos pulmonares ${ }^{1}$, su éxito se determina según la presencia o no de derrame sintomático o neumotórax, cuatro semanas después de la aplicación de la sustancia esclerosante ${ }^{5}$. Fue llevada a cabo por primera vez a inicios del siglo pasado por Spengler y posteriormente en 1935 por Bethune quien introdujo talco dentro de la cavidad pleural con el objetivo de colapsar el espacio residual existente posterior a una resección pulmonar ${ }^{6}$.

En la actualidad existen diversas sustancias utilizadas en dicho procedimiento dentro de ellas la Yodopovidona, un agente antiséptico tópico con capacidad de generar una respuesta inflamatoria potente gracias a sus propiedades citotóxicas y exudativas así como a su bajo $\mathrm{pH}^{7}$.

El propósito de este estudio es evaluar los resultados de la Yodopovidona como agente esclerosante al describir el porcentaje de derrame pleural o neumotórax espontáneo recidivante en sujetos mayores de 18 años con diagnóstico de derrame pleural de diversas etiologías y neumotórax espontáneo que fueron llevados a pleurodesis química con este agente en un Hospital Universitario de tercer nivel en Bogotá en el periodo comprendido entre enero de 2011 y mayo de 2014.

\section{MATERIALES Y MÉTODOS}

Se realizó un estudio observacional, descriptivo de series de casos en una población de 30 pacientes quienes presentaban derrame pleural y neumotórax espontáneo de diversas etiologías que fueron llevados a pleurodesis utilizando diferentes técnicas (pleurodesis química con bleomicina, pleurodesis química con yodopovidona y pleurodesis mecánica), durante el periodo comprendido entre Enero de 2011 y Mayo de 2014 en un Hospital Universitario de tercer nivel en Bogotá. Los criterios de inclusión fueron: Pacientes con diagnóstico de derrame pleural y neumotórax espontáneo, mayores de 18 años de edad que recibieron manejo con pleurodesis química con yodo. Del total de pacientes estudiados, 14 pacientes cumplían con los criterios de inclusión. Los 16 pacientes restantes fueron excluidos del estudio por ser menores de 18 años o haber sido tratados con técnicas de pleurodesis diferentes (mecánica) o usar sustancias diferentes a la yodopovidona.

Los datos se obtuvieron de la revisión sistemática de las historias clínicas y se tuvieron en cuenta variables como: sexo, edad, número de sujetos que presentan recidiva posterior a la realización del procedimiento, efectos adversos relacionados con el mismo (porcentaje de sangrado, porcentaje de dolor, porcentaje de fiebre y dificultad respiratoria), tiempo de retiro de los tubos de tórax posterior a la realización de pleurodesis y el promedio de días de hospitalización.

El análisis estadístico se efectuó mediante tablas de frecuencias, se calcularon medidas de tendencia central y porcentajes.

El seguimiento se realizó mediante la consulta de las historias clínicas con el fin de indagar la recurrencia de derrames o neumotórax un mes después de realizado el procedimiento. Los Criterios de Paladine $^{8}$ para evaluar la respuesta clínica a la pleurodesis se encuentran expuestos en la Tabla $\mathbf{1}$. La mayoría de los casos los derrames pleurales fueron clasificados bajo los criterios descritos por Light ${ }^{9}$; las muestras para esta clasificación fueron recopiladas mediante toracocentesis.

La instilación de yodo para la realización de pleurodesis se llevó a cabo mediante dos vías estandarizadas en los protocolos de atención del hospital: Drenaje pleural previamente instalado o toracoscopía, así mismo la solución instalada fue preparada de dos maneras: yodopovidona $20 \mathrm{~cm}^{3}$ + lidocaína o bupivacaina $20 \mathrm{~cm}^{3}$ más solución salina al $0,9 \% 60 \mathrm{~cm}^{3}$ para una mezcla total de 100 $\mathrm{cm}^{3}$ o Isodine solución + peróxido de hidrógeno.

\section{RESULTADOS}

Se analizaron un total de 14 pacientes, la mitad de ellos hombres. El promedio de edad fue de 61,85 años (Desviación Estándar de la población $(\sigma)$ : 13,87, Desviación Estándar de la muestra (S):14,39) con una mínima de 27 años y una máxima de 84 años.

Ocho pacientes presentaron derrame pleural maligno; los seis pacientes restantes presentaban otro tipo de patologías como: neumotórax espontáneo (4 pacientes), derrame pleural secundario a Síndrome de Alport (1 paciente) y derrame pleural subsiguiente a tuberculosis ( 1 paciente).

Una de las pacientes con cuadro de neumotórax 


\section{Tabla 1. Criterios de Paladine ${ }^{8}$ para evaluar la respuesta clinica a la pleuro- desis. \\ a) Respuesta completa: No existe acumulación de fluidos durante los primeros 30 días \\ b) Respuesta parcial: Recurrencia de una pequeña cantidad de derrame que no requieren drenaje \\ c) No respuesta: Recurrencia de derrame que necesita ser evacuado}

Tettey M, and Col. Tetracycline pleurodesis for malignant pleural effusion a review of 38 cases $^{8}$.

espontáneo presentaba como patología de base; carcinoma ductal infiltrante de mama izquierda metastásico a pulmón y hueso, los otros tres pacientes restantes que componen este grupo presentaban dicho hallazgo como consecuencia de enfermedad bullosa. La Figura 1 recopila las principales causas de derrame pleural maligno.

Dentro de los pacientes con derrame pleural maligno de origen pulmonar se establecieron diversos tipos los cuales se encuentran representados en la Figura 2.
De los 4 pacientes con neumotórax espontáneo, 2 fueron bilaterales, 1 derecho y 1 izquierdo, los pacientes con derrame pleural maligno 5 fueron derechos y 3 izquierdos, mientras que el paciente con derrame pleural secundario a Síndrome de Alport y la paciente con derrame secundario a tuberculosis presentaron derrames izquierdos; la Figura 3 compara la localización de los neumotórax con la totalidad de derrames pleurales. Todos los derrames pleurales a los que se les aplicó los criterios de Light

Figura 1. Causas del Derrame Pleural Maligno

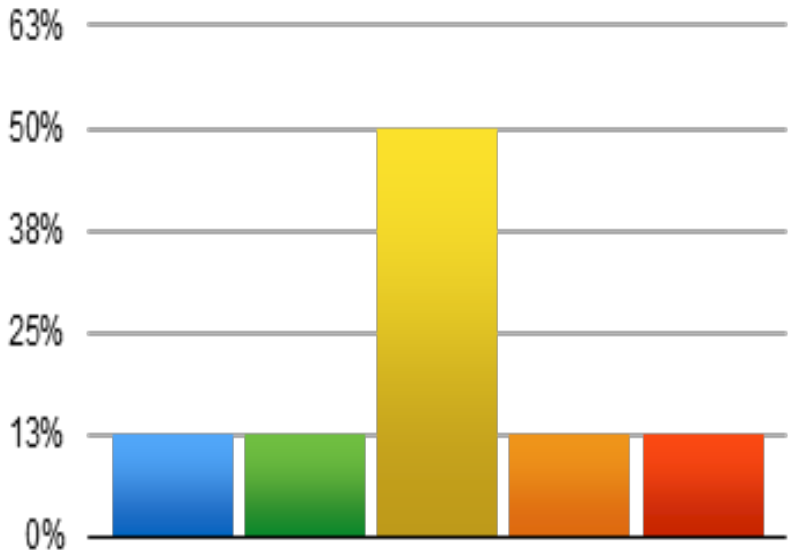

- Cancer de mama estadio IV metastásico a pleura

- Mesotelioma maligno

Cancer de origen pulmonar

Adenocarcinoma oculto

- Neoplasia mesenquimal pl eomórfica de tipo histiocitoma fibroso maligno metastásico a mama y pulmór

Fuente: Elaboración Propia. 
fueron clasificados como exudados. La instilación de yodo en 10 de los casos se realizó a través de drenaje pleural previamente instalado y 4 pacientes fueron llevados a realización de toracoscopía.

En 12 casos se administró Yodopovidona $20 \mathrm{~cm}^{3}$ más lidocaína o bupivacaina $20 \mathrm{~cm}^{3}$ más solución salina al $0,9 \% 60 \mathrm{~cm}^{3}$ para una mezcla total de 100 $\mathrm{cm}^{3}$, y en 2 casos se administró Isodine solución más peróxido de hidrógeno.

Una respuesta completa a la pleurodesis sin presencia de derrames pleurales recidivantes $o$ neumotórax posteriores al procedimiento en un seguimiento a un mes se presentó en 13 de los pacientes evaluados. Uno de los pacientes, con

Figura 2 . Tipos de Cancer Pulmonar y su distribución proporcional

= Cáncer pleuropulmonar sin clasificación histológica

- Adenocarcinoma metastásico a pleura y diafragma estadío IIIB/IV

- Tumor maligno de células grandes pleomórficas

Cáncer bronquio-alveolar derecho con compromiso pleural (presencia de células típicas de citoplasma amplio)

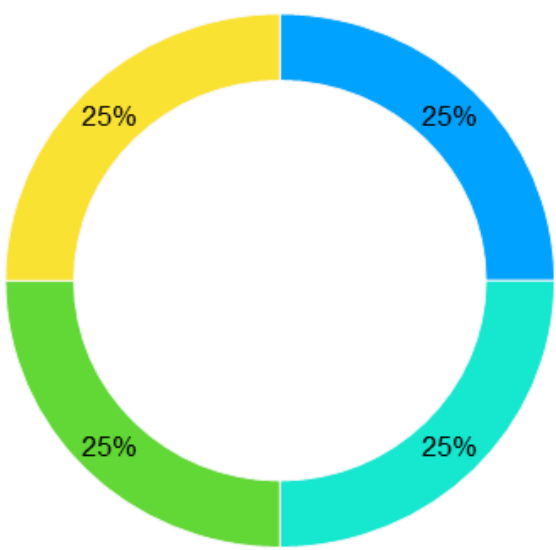

Figura 3 . Localización Neumotórax Vs Localización Derrame pleural

Derrame Pleural

Neumotórax
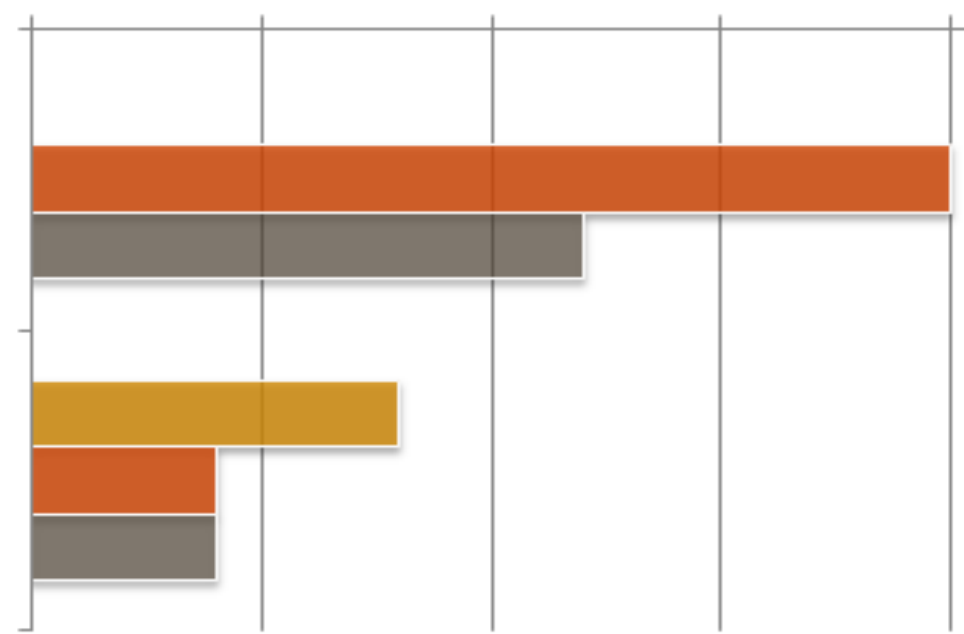

0

1

3

4

5 
diagnóstico de neumotórax espontáneo bilateral, debió ser llevado a una segunda pleurodesis con solución yodada por persistencia de neumotórax izquierdo posterior a la realización del procedimiento.

Solo se registró un fallecimiento durante la hospitalización, esta paciente tenía como diagnóstico neoplasia mesenquimal pleomórfica de tipo histiocitoma fibroso maligno metastásico a pulmón y mama, el fallecimiento se presentó 42 días después de su ingreso al hospital y 6 días posterior a la realización del procedimiento.

En cuanto a los efectos secundarios derivados de la instilación de Yodopovidona, se reportaron dos casos de dolor torácico leve, uno durante la realización del procedimiento y el otro dos días posteriores a la intervención, dicho síntoma fue controlado adecuadamente con la administración de opioides, en ningún caso se consignó presencias de dificultad respiratoria, fiebre, desarrollo de empiema o algún otro signo o síntoma. No se reportaron síntomas o signos de hipertiroidismo después de la ejecución del procedimiento, ni variación en los niveles de presión arterial. Después del procedimiento, no se midieron marcadores bioquímicos como pruebas de función hepática, PCR, pruebas de función renal, calcio, fosfatasa alcalina entre otros.

El promedio de estancia hospitalaria fue de 15 días, en la mayoría de los casos la prolongación de la estancia hospitalaria se debió al tratamiento de las patologías de base y no acomplicaciones derivadas del procedimiento o recidivas de derrames o neumotórax posterior a la pleurodesis. El promedio de días entre la inserción y el retiro del tubo del tórax fue de 4,1 días. El promedio de días transcurridos entre la realización de la pleurodesis y el retiro del tubo de tórax fue de 2,6 días.

\section{DISCUSIÓN}

La pleurodesis química es el procedimiento terapéutico menos invasivo y de elección para el manejo de pacientes con disnea secundaria a derrame pleural y neumotórax recurrente de cualquier etiología. Su objetivo es la obliteración del espacio pleural gracias a la respuesta biológica desencadenada por células mesoteliales que inducen inflamación difusa, desequilibrio entre coagulantes y fibrinolíticos, reclutamiento y proliferación de fibroblastos produciendo colágeno, citoquinas y factores de crecimiento que promueven la fibrosis del espacio pleural $^{10}$; esta respuesta genera una mejoría de la disnea y disminución de la recurrencia de derrames y neumotórax evitando las toracocentesis y toracostomías a repetición y así disminuyendo la morbilidad derivada de dichos procedimientos.

Se ha utilizado una amplia variedad de agentes esclerosantes pleurales sin hallazgo hasta el momento de un agente ideal que cumpla con características tales como: económico, de fácil consecución y capaz de producir una pleurodesis efectiva, eficaz y segura en corto tiempo de exposición ${ }^{11}$. La falta de respuesta a la administración de sustancias esclerosantes se debe a una inadecuada re-expansión pulmonar situación asociada a la presencia de una pleura visceral gruesa, loculaciones pleurales, presencia de obstrucción de las vías proximales y a la presencia de fugas permanentes ${ }^{12}$.

El Talco es uno de los agentes usados con más éxito alrededor del mundo, sin embargo, su uso se ha asociado con complicaciones graves como síndrome de dificultad respiratoria, neumonitis aguda, embolización sistémica y muerte, otros productos como la bleomicina son costosos y menos eficaces por lo que se usan con poca frecuencia ${ }^{13}$. La Yodopovidona es un antiséptico tópico utilizado para pleurodesis química por su capacidad de generar una intensa respuesta inflamatoria con fibrosis pleural ${ }^{12}$, es ampliamente absorbida por superficies mucosas así como por la glándula tiroides, puede aparecer en saliva, sudor y leche y es excretada casi sin cambios por la orina ${ }^{14}$.

En un estudio realizado en el Hospital Universitario de Alejandría ubicado al norte de Egipto, se reportó bajo riesgo de complicaciones graves tales como distrés respiratorio con el uso de Yodopovidona, efecto adverso que si es común al usar otro tipo de agentes como el talco ${ }^{15}$. El efecto adverso más frecuentemente relacionado con el uso de este compuesto es el dolor torácico, fiebre y empiema han sido mencionados en algunos estudios sin embargo han reportado baja incidencia ${ }^{16}$.

Existen reportes de disfunción transitoria de la función tiroidea, especialmente en infantes posterior a la aplicación tópica de Yodopovidona o al uso de medios de contraste a base de yodo, esto debido a que las altas concentraciones de yodo exógeno son capaces de bloquear la unión orgánica y las reacciones de acoplamiento disminuyendo la síntesis de hormonas tiroideas. En un estudio realizado en Turquía por Ali Yeginsu et al. ${ }^{17}$ no se evidenciaron signos y síntomas de hipo o hipertiroidismo, y no se encontraron diferencias estadísticamente significativas en los niveles de hormonas tiroideas antes y después de la pleurodesis.

Dentro de los efectos adversos relacionados con el uso de yodopovidona, Andrade et al. ${ }^{18}$ encontraron en su estudio que el dolor pleurítico es el efecto 
adverso más frecuente, seguido de variaciones en la medición de la presión arterial; otros efectos adversos incluyeron aumento en sangre de los niveles de Proteína $\mathrm{C}$ reactiva, fosfatasa alcalina, aspartato amino transferasa (AST) y alanina amino transferasa (ALT) así como un descenso de los niveles de sodio. Falla renal, confusión mental o pérdida de visión no fueron encontradas en este estudio ${ }^{18}$.

Segun el estudio realizado por Caglayan et al. ${ }^{4}$ en Turquía se encontró dolor torácico en el 16,2\% de los casos, fiebre en el 6,9\% y enfisema subcutáneo en dos casos.

Wagenfeld et al. ${ }^{19}$ informaron un reducción en la agudeza visual debido a la alteración del epitelio pigmentado de la retina en 3 pacientes que recibieron $10 \%$ de solución de Yodopovidona durante cirugía toracoscópica, sin embargo las dosis del agente esclerosante usadas fueron mucho mayores a las recomendadas.

Ibrahim islam et al. ${ }^{20}$ realizaron un estudio en el Hospital Universitario de Menoufia entre enero y noviembre de 2013, se tomaron pacientes con derrame pleural maligno que fueron sometidos a realización de pleurodesis, 21 con talco y 18 con Yodopovidona, se evidenció una respuesta completa al procedimiento con talco en el 71,4\% de los casos comparado con un $66,7 \%$ de los casos tratados con Yodopovidona, en cuanto a la presencia de dolor se evidencia un número menor de casos en los pacientes tratados con Yodopovidona en comparación de los tratados con talco, las tasas de fiebre fueron iguales en ambos grupos ${ }^{20}$

Olivares et al. $^{14}$ estudiaron pacientes de 14 hospitales de Tijuana y Ensenada en México con diagnóstico de derrame pleural maligno o recurrente que fueron llevados a pleurodesis química con yodopovidona, se observó una respuesta completa al manejo en 50 pacientes (96,1\%), el 5,8\% de los pacientes estudiados experimentaron dolor pleurítico o reacciones vasovagales durante o después del procedimiento ${ }^{14}$.

Entre los años 2015 y 2017 se realizó un ensayo clínico en el Hospital Ghaem de la cuidad de Mashhad en Irán, este comparó el efecto de la bleomicina versus la Yodopoviona en pleurodesis química, los investigadores determinaron que no había una diferencia significativa en cuanto a la respuesta al tratamiento o las complicaciones entre ambos agentes, sin embargo, señalan que la bleomicina es una agente más costoso y se recomienda su uso en pacientes con enfermedades pleurales no neoplásicas tales como neumotórax, insuficiencia cardiaca congestiva y cirrosis ${ }^{19}$.
En cuanto al neumotórax espontáneo, estudios como el realizado por ChengHung How et al. ${ }^{16}$ manifiestan tasas de recurrencia de neumotórax espontáneo posterior al uso de Yodopovidona del $0-6 \%$ los cuales son comparables con las reportadas para el talco (0-9\%). Por otra parte el estudio realizado por Estrada et al. ${ }^{21}$ en el Hospital de la Santa Creu I Sant Pau de Barcelona en el cual se incluyeron pacientes con neumotórax esponáneo que fueron llevados a realización de pleurodesis con Yodopovidona a través de videotoracoscopia, se reportó una tasa de recidiva de solo el 6,1\%, así como una adecuada tolerancia el procedimiento.

En un Hospital en Kolkata Singapur se estudiaron pacientes con diagnóstico de derrame pleural maligno o recurrente y neumotórax que fueron llevados a realización de pleurodesis con Yodopovidona, se evidenció una respuesta completa sin reacumulación de aire o fluido en $89,5 \%$ de los pacientes y presencia de efectos adversos; tales como dolor pleurítico intenso e hipotensión en solo el 7,9\% de los casos ${ }^{22}$.

Ziaoldin et al. $^{23}$ en su estudio determinaron que la respuestaalapleurodesiscon Yodopovidonanoguarda relación con el género o el hemitórax comprometido y no hay una relación estadísticamente significativa entre el tipo de malignidad y la recurrencia de derrame pleural.

Dentro de las debilidades del presente estudio se incluyen: la baja población incluida, esto debido a la poca frecuencia de realización del procedimiento por parte del Servicio de Cirugía de Tórax de este hospital, además existen pacientes que aunque fueron sometidos al procedimiento no pudieron ser reclutados para este estudio, no se realizó la respectiva descripción quirúrgica y por tanto no fueron localizables en las bases de datos revisadas.

En conclusión, la pleurodesis química con Yodopovidona es una alternativa segura, fácilmente disponible y de bajo costo para el tratamiento de derrame pleural y neumotórax espontaneo de diversas etiologías, generando buenos resultados, bajas tasas de recidiva y escasos efectos adversos razón por la cual se considera se puede estandarizar su uso en los servicios de Cirugía de Tórax de todos los hospitales a nivel nacional e internacional. 


\section{REFERENCIAS}

1. European Lung Foundation. Neumotórax espontáneo primario (NEP). Disponible en: https://www. europeanlung.org/assets/files/es/publications/psp-spanish. $p d f$

2. Rivas J, Canalís E, Molins L, Pac J, Torres A, Torres J; Grupo de Trabajo SEPAR. Normativa sobre diagnóstico y tratamiento del neumotórax. Arch Bronconeumol 2002; 38: 589-95. Disponible en: https:// www.archbronconeumol.org/es-pdf-S0300289602752964

3. Bejarano M. Derrames pleurales en urgencias; Rev Colomb Cir.1999;14(4):263-273. Disponible en: https:// encolombia.com/medicina/revistas-medicas/cirugia/vc-144/ cirugia14499-revision-derrames/

4. Kahrom H, Aghajanzadeh M, Reza M, Kahrom M. Efficacy and safety of povidone-iodine pleurodesis in malignant pleural effusions. Indian J of Palliat Care. 2017;23(1):53-56. Disponible en: https://www.ncbi.nlm. nih.gov/pmc/articles/PMC5294438/

5. British Thoracic Society. BTS Pleural Disease Guideline 2010. Thorax 2010.;65 (Suppl 2):II1-II3. Disponible en: http://www.sppneumologia.pt/uploads/ files/comissoestrabalho/ct\%20Tecnicas\%20Endoscopicas/ PDF3.pdf

6. Costa M, Marchi E, Suso F. Pleurodesis: technique and indications. J Bras Pneumol. 2006; 32(4):347-356. Disponible en: http://www.scielo.br/scielo.php?script=sci_ar ttextépid=S1806-37132006000400014

7. Agarwal R, Aggarwal A, Gupta D, Jindal S. Efficacy and safety of iodopovidone in chemical pleurodesis: A meta-analysis of observational studies. Respir Med .2006;100:2043-2047. Disponible en: https://www. sciencedirect.com/science/article/pii/S0954611106000941

8. Tettey M, Sereboe L, Edwin F, Frimpong-boateng K. Tetracycline pleurodesis for malignant pleural effusion a review of 38 cases. Ghana Med J. 2005;32:128-131. Disponible en: https://www.ncbi.nlm.nih.gov/pmc/articles/ PMC1790826/

9. Paez N. Guías de atención clínica derrame pleural. Fundacion Neumologica Colombiana.2006. Disponible en: https://s3.amazonaws.com/academia.edu. documents/39042932/DERRAME_PLEURAL_GPC.pdf? AWSAccessKeyId=AKIAIWOWYYG Z2Y53UL $\overline{3} A$ \& Expire $s=1557761336 \&$ Signature $=O O$ iXFo $21 \mathrm{Hd} 7 \mathrm{~h} 1 \mathrm{REecNAOH}$ fyUtbA\%3D \& response-content-disposition=inline $\% 3 B \% 20$ filename\%3DDERRAME_PLEU RAL_GPC.pdf

10. Mohamed K, Hassan O. A new look at an old agent for pleurodesis. Egy J chest Dis \& Tub. 2013; 62:617620.2222. Disponible en: https://core.ac.uk/download/ pdf/82552083.pdf

11. Ghoneim A, Elkomy H, Elshora A, Mehrez M. Usefulness of pigtail catheter in pleurodesis of malignant pleural effusion. Egy J chest Dis \& Tub. 2014; 63:107-112. Disponible en: https://www.sciencedirect. com/science/article/pii/S0422763813002616 12. Sheata S, Sileem A,El-Fakharany K. Pleural fluid CRP, $\mathrm{LDH}$, and $\mathrm{pH}$ as predictors of successful pleurodesis in malignant pleural effusions. Egy J chest Dis \& Tub .2015; 64:593-599. Disponible en: https://core.ac.uk/download/ $p d f / 82183561 . p d f$
13. Pandya H, Kapur M. Role of povidone-iodine as an effective and safe agent in chemical pleurodesis. Int $J$ Sci Res. 2018; 7(4):49-50. Disponible en: https://wwjournals. com/index.php/ijsr/article/view/3328

14. Olivares C, Laniado R, Leon C, Reyes A, Chavez C, Ligth R. Iodopovidone Pleurodesis for Recurrent Pleural Effusions. Chest. 2002;122:581-583. Disponible en: https://www.ncbi.nlm.nih.gov/pubmed/12171835

15. Mohamed E, Abd Alla A. Thoracoscopic pleurodesis using iodopovidone versus pleural abrasions in management of recurrent pneumothorax. Egy J chest

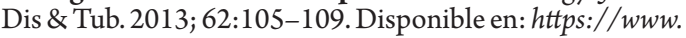
sciencedirect.com/science/article/pii/S0422763813000058

16. How C, Hsu H, Chen J. Chemical pleurodesis for spontaneous Pneumothorax. J Formos Med Assoc.2013; 112:749e-55. Disponible en: https://www.ncbi.nlm.nih. gov/pubmed/24268613

17. Yeginsu A, Karamustafaoglu A, Ozugurlu F, Etikan L. Iodopovidone pleurodesis not effect thyroid function in normal adults. Interact Cardiovasc Thorac Surg. 2007; 6:563-564. Disponible en:https://www.ncbi.nlm.nih.gov/ pubmed/17669938

18. Andrade J, Mingarini R, Maia R, Vianna S, Pego P. Safety profile of the use of iodopividone for pleurodesis in patients with malignant pleural effusion. Respiration. 2015; 90:369-375. Disponible en: https://www.ncbi.nlm. nih.gov/pubmed/26439936

19. Bagheri R, Noori M, Rajayi M, Attaran D, Hashem A, Mohammadzadeh S, Basiri R, Rezaeetalab F,Afghani $R$, Salehi $M$. The effect of vidone versus bleomycin in chemical pleurodesis. Asian Cardiovasc Thorac Ann. 2018; 26(5): 382-386. Disponible en: https://journals. sagepub.com/doi/abs/10.1177/0218492318778485

20. Ibrahim I, Dokhan A, El-Sessy A, Eltawee M. Povidone-iodine pleurodesis versus talc pleurodesis in preventing recurrence of malignant pleural effusion. J Cardiothorac Surg. 2015;10:64. Disponible en: https:// www.ncbi.nlm.nih.gov/pubmed/25947235

21. Estrada S, Ríos C, Alfara J, Fibla A, Gómez S, Leon C. Spontaneous pneumothorax: pleurodesis with an iodopovidone hydroalcoholic solution. Arch Bronconeumol. 2003;39(4):171-4. Disponible en: https://www.ncbi.nlm. nih.gov/pubmed/12716558

22. Dey A, Bhuniya S, Datta A, Pandit S, Saha-Dutta M, Sengupta A, Saha I. Iodopovidone pleurodesis: experience of a tertiary hospital in Kolkata. Singapore Med J. 2010;51(2): 163-5. Disponible en: https://www. ncbi.nlm.nih.gov/pubmed/20358157

23. RasiHashemi S, Ramouz A. Effect of Betadine in chemical pleurodesis in patients with recurrent malignant pleural effusion between 2013 and 2015. J surgery and trauma. 2017;5(1-2):7-12. Disponible en: https://pdfs.semanticscholar.org/b399/92a1075c20afb8626 $6 b 46 d 2 f c 89 b 5 d 583 f 3 a . p d f$ 